\title{
Fuzzy Anti-n-Continuous and n-Bounded Linear Operators
}

\author{
B. Surender Reddy ${ }^{1} \&$ M. Srinivas ${ }^{1}$ \\ ${ }^{1}$ Department of Mathematics, University College of Science, Saifabad, Osmania University, Hyderabad, AP, India \\ Correspondence: B. Surender Reddy, Department of Mathematics, University College of Science, Saifabad, Os- \\ mania University, Hyderabad, AP, India. E-mail: bsrmathou@ yahoo.com
}

Received: September 16, 2013 Accepted: October 7, 2013 Online Published: November 14, 2013

doi:10.5539/jmr.v5n4p71 URL: http://dx.doi.org/10.5539/jmr.v5n4p71

\begin{abstract}
In this paper we study the concept of Fuzzy-anti-n-normed linear operator as a generalization of Fuzzy-anti-2normed linear operator. Fuzzy-anti-n-continuous linear operator and three types (strongly, weakly, and sequentially) of Fuzzy-anti-n-continuous linear operators are defined and relation between strongly, weakly and sequentially Fuzzy-anti-n-continuous linear operator is developed. Also strongly and weakly fuzzy-anti n-bounded linear operators are defined and relation between Fuzzy-anti-n-continuous linear operator and Fuzzy-anti-n-bounded linear operators is established.
\end{abstract}

Keywords: fuzzy-anti-n-linear operator, fuzzy-anti-n-continuous-linear operator, strongly, weakly, sequentially fuzzy-anti-n-continuous-linear operators, fuzzy-anti-n-bounded-linear operators

\section{Introduction}

The idea of Fuzzy norm was initiated by Katsaras (1984). In 1993, Felbin introduced an idea of Fuzzy norm on a linear space by assigning a Fuzzy Real number to each element of the linear space, so that the corresponding metric associated this Fuzzy norm is a Kaleva type fuzzy metric. Narayanan and Vijayabalaji (2005) extended the notion of n-normed linear space to fuzzy-n-normed-linear space. In 2010, Jebril and Samanta introduced fuzzy-antinorm on a linear space depending on the idea of fuzzy-anti-norm was introduced by Bag and Samanta (2003) and investigated their important properties. In 2011, Reddy studied fuzzy-anti-2-norm and some results are established in fuzzy-anti-2-normed linear space and Reddy (2011) introduced fuzzy-anti-n-norm on linear space and studied the notion of convergent sequence, Cauchy sequence in fuzzy-anti-n-normed linear space. Sinha, Mishra, Lal (2011, 2012) introduced the concept of fuzzy-anti-2-continous linear operator and fuzzy-anti-2-bounded linear operator on fuzzy-anti-2-normed linear space. In this paper we introduced the concept of fuzzy-anti-n-continuous linear operator on a fuzzy-anti-n-normed linear space to another fuzzy-anti-n-normed linear space and defined three types (strongly, weakly and sequentially) of fuzzy-anti-n-continuous linear operators and relation between strongly, weakly and sequentially fuzzy-anti-n-continuous linear operator is developed. Also introduced the concept of fuzzy-anti-n-bounded linear operator on a fuzzy-anti-n-normed linear space to another fuzzy-anti n-normed linear space and defined two types (strongly ane weakly) of fuzzy-anti-n-bounded linear operators and relation between strongly, weakly fuzzy-anti-n-bounded linear operator is established.

\section{Preliminaries}

This section contains a few basic definitions and preliminary results which will be needed in the sequel.

Definition 2.1 Let $n \in N$ and let $X$ be a real linear space of dimension $d \geq n$. A real valued function $\|\bullet, \bullet, \ldots, \bullet\|$ : $X \times X \times \ldots \times X \rightarrow R$ satisfying the following four properties

$n N_{1}:\left\|x_{1}, x_{2}, \ldots, x_{n}\right\|=0$ if and only if $x_{1}, x_{2}, \ldots, x_{n}$ are linearly dependent vectors.

$n N_{2}:\left\|x_{1}, x_{2}, \ldots, x_{n}\right\|=\left\|x_{j_{1}}, x_{j_{2}}, \ldots, x_{j_{n}}\right\|$ for every permutation $\left(j_{1}, j_{2}, \ldots, j_{n}\right)$ of $(1,2, \ldots, n)$, i.e., $\left\|x_{1}, x_{2}, \ldots, x_{n}\right\|$ is invariant under any permutation of $x_{1}, x_{2}, \ldots, x_{n}$.

$n N_{3}:\left\|x_{1}, x_{2}, \ldots, x_{n-1}, \alpha x_{n}\right\|=|\alpha|\left\|x_{1}, x_{2}, \ldots, x_{n}\right\|$ for all $\alpha \in R$.

$n N_{4}:\left\|x_{1}, x_{2}, \ldots, x_{n-1}, y+z\right\| \leq\left\|x_{1}, x_{2}, \ldots, x_{n-1}, y\right\|+\left\|x_{1}, x_{2}, \ldots, x_{n-1}, z\right\|$ for all $y, z, x_{1}, x_{2}, \ldots, x_{n-1} \in X$, is called an n-norm on $X$ and the pair $(X,\|\bullet, \bullet, \ldots, \bullet\|)$ is called $n$-normed linear space. 
Definition 2.2 Let $X$ be a linear space over a real field $F$. A fuzzy subset $N$ of $X \times X \times \ldots \times X \times R \rightarrow R$ is called a fuzzy n-norm on $X$ if the following conditions are satisfied for all $x_{1}, x_{2}, \ldots, x_{n}, x_{n}^{\prime} \in X$ and

$(n-N 1)$ : For all $t \in R$ with $t \leq 0, N\left(x_{1}, x_{2}, \ldots, x_{n}, t\right)=0$.

$(n-N 2)$ : For all $t \in R$ with $t>0, N\left(x_{1}, x_{2}, \ldots, x_{n}, t\right)=1$ if and only if $x_{1}, x_{2}, \ldots, x_{n}$ are linearly dependent.

$(n-N 3): N\left(x_{1}, x_{2}, \ldots, x_{n}, t\right)$ is invariant under any permutation of $x_{1}, x_{2}, \ldots, x_{n}$.

$(n-N 4)$ : For all $t \in R$ with $t>0, N\left(x_{1}, x_{2}, \ldots, x_{n-1}, c x_{n}, t\right)=N\left(x_{1}, x_{2}, \ldots, x_{n}, \frac{t}{|c|}\right)$ if $c \neq 0, c \in F$.

$(n-N 5): \forall s, t \in R$,

$$
N\left(x_{1}, x_{2}, \ldots, x_{n-1}, x_{n}+x_{n}^{\prime}, s+t\right) \geq \min \left\{N\left(x_{1}, x_{2}, \ldots, x_{n-1}, x_{n}, s\right), N\left(x_{1}, x_{2}, \ldots, x_{n-1}, x_{n}^{\prime}, t\right)\right\}
$$

$(n-N 6): N\left(x_{1}, x_{2}, \ldots, x_{n}, t\right)$ is a non-decreasing function of $t \in R$ and $\lim _{t \rightarrow \infty} N\left(x_{1}, x_{2}, \ldots, x_{n}, t\right)=1$.

Then $N$ is said to be a fuzzy n-norm on a linear space $X$ and the pair $(X, N)$ is said to be a fuzzy n-normed linear space (briefly F-n-NLS).

The following condition of fuzzy n-norm $N$ will be required later on $(n-N 7)$ : For all $t \in R$ with $t>0, N\left(x_{1}, x_{2}, \ldots, x_{n}, t\right)>0$, implies that $x_{1}, x_{2}, \ldots, x_{n}$ are linearly dependent.

Definition 2.3 Let $X$ be a linear space over a real field $F$. A fuzzy subset $N^{*}$ of $X \times X \times \ldots \times X \times R \rightarrow R$ such that for all $x_{1}, x_{2}, \ldots, x_{n}, x_{n}^{\prime} \in X$ and $c \in F$

$\left(n-N^{*} 1\right)$ : For all $t \in R$ with $t \leq 0, N^{*}\left(x_{1}, x_{2}, \ldots, x_{n}, t\right)=1$.

$\left(n-N^{*} 2\right)$ : For all $t \in R$ with $t>0, N^{*}\left(x_{1}, x_{2}, \ldots, x_{n}, t\right)=0$ if and only if $x_{1}, x_{2}, \ldots, x_{n}$ are linearly dependent.

$\left(n-N^{*} 3\right): N^{*}\left(x_{1}, x_{2}, \ldots, x_{n}, t\right)$ is invariant under any permutation of $x_{1}, x_{2}, \ldots, x_{n}$.

$\left(n-N^{*} 4\right)$ : For all $t \in R$ with $t>0, N^{*}\left(x_{1}, x_{2}, \ldots, c x_{n}, t\right)=N^{*}\left(x_{1}, x_{2}, \ldots, x_{n}, \frac{t}{|c|}\right)$ if $c \neq 0$.

$\left(n-N^{*} 5\right)$ : For all $s, t \in R$,

$$
N^{*}\left(x_{1}, x_{2}, \ldots, x_{n-1}, x_{n}+x_{n}^{\prime}, s+t\right) \leq \max \left\{N^{*}\left(x_{1}, x_{2}, \ldots, x_{n-1}, x_{n}, s\right), N^{*}\left(x_{1}, x_{2}, \ldots, x_{n-1}, x_{n}^{\prime}, t\right)\right\} .
$$

$\left(n-N^{*} 6\right): N^{*}\left(x_{1}, x_{2}, \ldots, x_{n}, t\right)$ is a non-increasing function of $t \in R$ and

$$
\lim _{t \rightarrow \infty} N^{*}\left(x_{1}, x_{2}, \ldots, x_{n}, t\right)=0 .
$$

Then $N^{*}$ is said to be a fuzzy anti-n-norm on a linear space $X$ and the pair $\left(X, N^{*}\right)$ is called a fuzzy anti-n-normed linear space (briefly Fa-n-NLS).

The following condition of fuzzy anti-n-norm $N^{*}$ will be required later on.

$\left(n-N^{*} 7\right)$ : For all $t \in R$ with $t>0, N^{*}\left(x_{1}, x_{2}, \ldots, x_{n}, t\right)<1$, implies that $x_{1}, x_{2}, \ldots, x_{n}$ are linearly dependent.

\section{Fuzzy Anti n-Continuous Linear Operators}

Let $\left(X, N_{1}^{*}\right)$ and $\left(Y, N_{2}^{*}\right)$ are fuzzy-anti-n-normed-linear spaces defined on the same field.

Definition 3.1 $T$ is a mapping from $X_{1} \times X_{2} \times \ldots \times X_{n}$ to $Y_{1} \times Y_{2} \times \ldots \times Y_{n}$ where $X_{1}, X_{2}, \ldots, X_{n}$ and $Y_{1}, Y_{2}, \ldots, Y_{n}$ are subspaces of $\left(X, N_{1}^{*}\right),\left(Y, N_{2}^{*}\right)$ respectively. Then $T$ is said to be fuzzy-anti-n-linear operator, if

$$
T\left(\sum_{i_{n}=1}^{n} x_{1}^{\left(i_{n}\right)}, \sum_{i_{n-1}=1}^{n} x_{2}^{\left(i_{n-1}\right)}, \sum_{i_{n-2}=1}^{n} x_{3}^{\left(i_{n-2}\right)}, \ldots ., \sum_{i_{2}=1}^{n} x_{n-1}^{\left(i_{2}\right)}, \sum_{i_{1}=1}^{n} x_{n}^{\left(i_{1}\right)}\right)=\sum_{i_{1}=1}^{n} \sum_{i_{2}=1}^{n} \sum_{i_{3}=1}^{n} \ldots \sum_{i_{n}=1}^{n} T\left(x_{1}^{\left(i_{n}\right)}, x_{2}^{\left(i_{n-1}\right)}, x_{3}^{\left(i_{n-2}\right)}, \ldots ., x_{n-1}^{\left(i_{2}\right)}, x_{n}^{\left(i_{1}\right)}\right)
$$

and

$$
T\left(\alpha_{1} x_{1}, \alpha_{2} x_{2}, \ldots, \alpha_{n} x_{n}\right)=\alpha_{1} \alpha_{2} \ldots \alpha_{n} T\left(x_{1}, x_{2}, \ldots, x_{n}\right), \forall\left(x_{1}, x_{2}, \ldots, x_{n}\right) \in X_{1} \times X_{2} \times \ldots \times X_{n} .
$$

Definition 3.2 Let $T$ be a fuzzy-anti-n-linear map from $X_{1} \times X_{2} \times \ldots \times X_{n}$ to $Y_{1} \times Y_{2} \times \ldots \times Y_{n}, X_{1}, X_{2}, \ldots, X_{n}$ and $Y_{1}, Y_{2}, \ldots, Y_{n}$ are subspaces of $\left(X, N_{1}^{*}\right),\left(Y, N_{2}^{*}\right)$ respectively. Then $T$ is called fuzzy-anti-n-continuous at $\left(x_{0}^{(1)}, x_{0}^{(2)}, x_{0}^{(3)}\right.$, $\left.\ldots, x_{0}^{(n)}\right) \in X_{1} \times X_{2} \times \ldots \times X_{n}$ if given $\varepsilon>0, \alpha \in(0,1) \exists \delta=\delta(\alpha, \varepsilon)>0, \beta=\beta(\alpha, \varepsilon) \in(0,1)$, such that for all $\left(x^{(1)}, x^{(2)}, x^{(3)}, \ldots, x^{(n)}\right) \in X_{1} \times X_{2} \times \ldots \times X_{n}$

$$
N_{1}^{*}\left[\left(x^{(1)}, x^{(2)}, x^{(3)}, \ldots, x^{(n)}\right)-\left(x_{0}^{(1)}, x_{0}^{(2)}, x_{0}^{(3)}, \ldots, x_{0}^{(n)}\right), \delta\right]<\beta
$$




$$
\Rightarrow N_{2}^{*}\left[T\left(x^{(1)}, x^{(2)}, x^{(3)}, \ldots, x^{(n)}\right)-T\left(x_{0}^{(1)}, x_{0}^{(2)}, x_{0}^{(3)}, \ldots, x_{0}^{(n)}\right), \varepsilon\right]<\alpha .
$$

If $T$ is fuzzy-anti-n-continuous at every point of $T: X_{1} \times X_{2} \times \ldots \times X_{n} \rightarrow Y_{1} \times Y_{2} \times \ldots \times Y_{n}$ then $T$ is fuzzy-anti-ncontinuous on $X_{1} \times X_{2} \times \ldots \times X_{n}$.

From now we will denote fuzzy-anti-n-continuous map by fa-n-continuous map.

Definition 3.3 Let $T: X_{1} \times X_{2} \times \ldots \times X_{n} \rightarrow Y_{1} \times Y_{2} \times \ldots \times Y_{n}$ be a fuzzy-anti-n-linear mapping, $X_{1}, X_{2}, \ldots, X_{n}$ and $Y_{1}, Y_{2}, \ldots, Y_{n}$ are subspaces of $\left(X, N_{1}^{*}\right),\left(Y, N_{2}^{*}\right)$ respectively. Then $T$ is called sequentially-fuzzy-anti-n-continuous at $\left(x_{0}^{(1)}, x_{0}^{(2)}, x_{0}^{(3)}, \ldots, x_{0}^{(n)}\right) \in X_{1} \times X_{2} \times \ldots \times X_{n}$ if

$$
\begin{aligned}
\forall k,\left(x_{k}^{(1)}, x_{k}^{(2)}, x_{k}^{(3)}, \ldots, x_{k}^{(n)}\right) \rightarrow\left(x_{0}^{(1)}, x_{0}^{(2)}, x_{0}^{(3)}, \ldots, x_{0}^{(n)}\right) \\
\Rightarrow T\left(x_{k}^{(1)}, x_{k}^{(2)}, x_{k}^{(3)}, \ldots, x_{k}^{(n)}\right) \rightarrow T\left(x_{0}^{(1)}, x_{0}^{(2)}, x_{0}^{(3)}, \ldots, x_{0}^{(n)}\right) .
\end{aligned}
$$

i.e.

$$
\begin{aligned}
& \lim _{k \rightarrow \infty} N_{1}^{*}\left[\left(x_{k}^{(1)}, x_{k}^{(2)}, x_{k}^{(3)}, \ldots, x_{k}^{(n)}\right)-\left(x_{0}^{(1)}, x_{0}^{(2)}, x_{0}^{(3)}, \ldots, x_{0}^{(n)}\right), t\right]=0, \forall t>0 \\
\Rightarrow & \lim _{k \rightarrow \infty} N_{2}^{*}\left[T\left(x_{k}^{(1)}, x_{k}^{(2)}, x_{k}^{(3)}, \ldots, x_{k}^{(n)}\right)-T\left(x_{0}^{(1)}, x_{0}^{(2)}, x_{0}^{(3)}, \ldots, x_{0}^{(n)}\right), t\right]=0, \forall t>0 .
\end{aligned}
$$

From now we will denote sequentially-fuzzy-anti-n-continuous map by Sq-fa-n-continuous map.

If $T$ is Sq-fa-n-continuous at every point of $X_{1} \times X_{2} \times \ldots \times X_{n}$ then $T$ is called Sq-fa-n-continuous on $X_{1} \times X_{2} \times \ldots \times X_{n}$.

Definition 3.4 Let $T: X_{1} \times X_{2} \times \ldots \times X_{n} \rightarrow Y_{1} \times Y_{2} \times \ldots \times Y_{n}$ be a fuzzy-anti-n-linear mapping, $X_{1}, X_{2}, \ldots, X_{n}$ and $Y_{1}, Y_{2}, \ldots, Y_{n}$ are subspaces of $\left(X, N_{1}^{*}\right),\left(Y, N_{2}^{*}\right)$ respectively. Then $T$ is called strongly-fuzzy-anti-n-continuous at $\left(x_{0}^{(1)}, x_{0}^{(2)}, x_{0}^{(3)}, \ldots, x_{0}^{(n)}\right) \in X_{1} \times X_{2} \times \ldots \times X_{n}$, if for each $\varepsilon>0, \exists \delta>0$ such that $\forall\left(x^{(1)}, x^{(2)}, x^{(3)}, \ldots, x^{(n)}\right) \in$ $X_{1} \times X_{2} \times \ldots \times X_{n}$,

$$
\begin{aligned}
& N_{2}^{*}\left[T\left(x^{(1)}, x^{(2)}, x^{(3)}, \ldots, x^{(n)}\right)-T\left(x_{0}^{(1)}, x_{0}^{(2)}, x_{0}^{(3)}, \ldots, x_{0}^{(n)}\right), \varepsilon\right] \\
& \leq N_{1}^{*}\left[\left(x^{(1)}, x^{(2)}, x^{(3)}, \ldots, x^{(n)}\right)-\left(x_{0}^{(1)}, x_{0}^{(2)}, x_{0}^{(3)}, \ldots, x_{0}^{(n)}\right), \delta\right] .
\end{aligned}
$$

From now we will denote strongly-fuzzy-anti-n-continuous map by St-fa-n-continuous map.

Definition 3.5 Let $T: X_{1} \times X_{2} \times \ldots \times X_{n} \rightarrow Y_{1} \times Y_{2} \times \ldots \times Y_{n}$ be fuzzy-anti-n-linear mapping, $X_{1}, X_{2}, \ldots, X_{n}$ and $Y_{1}, Y_{2}, \ldots, Y_{n}$ are subspaces of $\left(X, N_{1}^{*}\right),\left(Y, N_{2}^{*}\right)$ respectively. Then $T$ is called weakly-fuzzy-anti-n-continuous at $\left(x_{0}^{(1)}, x_{0}^{(2)}, x_{0}^{(3)}, \ldots, x_{0}^{(n)}\right) \in X_{1} \times X_{2} \times \ldots \times X_{n}$, if for a given $\varepsilon>0, \alpha \in(0,1), \exists \delta=\delta(\alpha, \varepsilon)>0$, such that $\forall\left(x^{(1)}, x^{(2)}, x^{(3)}, \ldots, x^{(n)}\right) \in X_{1} \times X_{2} \times \ldots \times X_{n}$,

$$
\begin{aligned}
& N_{1}^{*}\left[\left(x^{(1)}, x^{(2)}, x^{(3)}, \ldots, x^{(n)}\right)-\left(x_{0}^{(1)}, x_{0}^{(2)}, x_{0}^{(3)}, \ldots, x_{0}^{(n)}\right), \delta\right] \leq 1-\alpha \\
\Rightarrow & N_{2}^{*}\left[T\left(x^{(1)}, x^{(2)}, x^{(3)}, \ldots, x^{(n)}\right)-T\left(x_{0}^{(1)}, x_{0}^{(2)}, x_{0}^{(3)}, \ldots, x_{0}^{(n)}\right), \varepsilon\right] \leq 1-\alpha .
\end{aligned}
$$

From now we will denote weakly-fuzzy-anti-n-continuous map by Wk-fa-n-continuous map.

Theorem 3.6 Let $T: X_{1} \times X_{2} \times \ldots \times X_{n} \rightarrow Y_{1} \times Y_{2} \times \ldots \times Y_{n}$ be a fuzzy-anti-n-linear mapping, $X_{1}, X_{2}, \ldots, X_{n}$ and $Y_{1}, Y_{2}, \ldots, Y_{n}$ are subspaces of $\left(X, N_{1}^{*}\right),\left(Y, N_{2}^{*}\right)$ respectively. If $T$ is $S t$-fa- $n$-continuous then $T$ is $S q$-fa- $n$-continuous.

Proof. Let us assume that $T$ is St-fa-n-continuous at $\left(x_{0}^{(1)}, x_{0}^{(2)}, x_{0}^{(3)}, \ldots, x_{0}^{(n)}\right) \in X_{1} \times X_{2} \times \ldots \times X_{n}$, then for each $\varepsilon>0$, $\exists \delta=\delta\left(x_{0}^{(1)}, x_{0}^{(2)}, x_{0}^{(3)}, \ldots, x_{0}^{(n-1)}, x_{0}^{(n)}, \varepsilon\right)>0$, such that for all $\left(x^{(1)}, x^{(2)}, x^{(3)}, \ldots, x^{(n)}\right) \in X_{1} \times X_{2} \times \ldots \times X_{n}$,

$$
\begin{aligned}
& N_{2}^{*}\left[T\left(x^{(1)}, x^{(2)}, x^{(3)}, \ldots, x^{(n)}\right)-T\left(x_{0}^{(1)}, x_{0}^{(2)}, x_{0}^{(3)}, \ldots, x_{0}^{(n)}\right), \varepsilon\right] \\
& \leq N_{1}^{*}\left[\left(x^{(1)}, x^{(2)}, x^{(3)}, \ldots, x^{(n)}\right)-\left(x_{0}^{(1)}, x_{0}^{(2)}, x_{0}^{(3)}, \ldots, x_{0}^{(n)}\right), \delta\right]
\end{aligned}
$$

Let $\left(x_{k}^{(1)}, x_{k}^{(2)}, x_{k}^{(3)}, \ldots, x_{k}^{(n)}\right)$ be a sequence in $X_{1} \times X_{2} \times \ldots \times X_{n}$, such that

$$
\left(x_{k}^{(1)}, x_{k}^{(2)}, x_{k}^{(3)}, \ldots, x_{k}^{(n)}\right) \rightarrow\left(x_{0}^{(1)}, x_{0}^{(2)}, x_{0}^{(3)}, \ldots, x_{0}^{(n)}\right)
$$

i.e.,

$$
\lim _{k \rightarrow \infty} N_{1}^{*}\left[\left(x_{k}^{(1)}, x_{k}^{(2)}, x_{k}^{(3)}, \ldots, x_{k}^{(n)}\right)-\left(x_{0}^{(1)}, x_{0}^{(2)}, x_{0}^{(3)}, \ldots, x_{0}^{(n)}\right), t\right]=0, \forall t>0 .
$$


Now from Equation (1), by (2) we have

$$
\begin{gathered}
N_{2}^{*}\left[T\left(x_{k}^{(1)}, x_{k}^{(2)}, x_{k}^{(3)}, \ldots, x_{k}^{(n)}\right)-T\left(x_{0}^{(1)}, x_{0}^{(2)}, x_{0}^{(3)}, \ldots, x_{0}^{(n)}\right), \varepsilon\right] \leq N_{1}^{*}\left[\left(x_{k}^{(1)}, x_{k}^{(2)}, x_{k}^{(3)}, \ldots, x_{k}^{(n)}\right)-\left(x_{0}^{(1)}, x_{0}^{(2)}, x_{0}^{(3)}, \ldots, x_{0}^{(n)}\right), \delta\right] \\
\Rightarrow \lim _{k \rightarrow \infty} N_{2}^{*}\left[T\left(x_{k}^{(1)}, x_{k}^{(2)}, \ldots, x_{k}^{(n)}\right)-T\left(x_{0}^{(1)}, x_{0}^{(2)}, \ldots, x_{0}^{(n)}\right), \varepsilon\right] \leq \lim _{k \rightarrow \infty} N_{1}^{*}\left[\left(x_{k}^{(1)}, x_{k}^{(2)}, \ldots, x_{k}^{(n)}\right)-\left(x_{0}^{(1)}, x_{0}^{(2)}, \ldots, x_{0}^{(n)}\right), \delta\right] \\
\Rightarrow \lim _{k \rightarrow \infty} N_{2}^{*}\left[T\left(x_{k}^{(1)}, x_{k}^{(2)}, x_{k}^{(3)}, \ldots, x_{k}^{(n)}\right)-T\left(x_{0}^{(1)}, x_{0}^{(2)}, x_{0}^{(3)}, \ldots, x_{0}^{(n)}\right), \varepsilon\right]=0 .
\end{gathered}
$$

Since $\varepsilon$ is arbitrarily small positive real, it immediately follows that $T\left(x_{k}^{(1)}, x_{k}^{(2)}, x_{k}^{(3)}, \ldots, x_{k}^{(n)}\right) \rightarrow T\left(x_{0}^{(1)}, x_{0}^{(2)}, x_{0}^{(3)}, \ldots\right.$, $\left.x_{0}^{(n)}\right)$. Therefore $T$ is Sq-fa-n-continuous.

Theorem 3.7 Let $T: X_{1} \times X_{2} \times \ldots \times X_{n} \rightarrow Y_{1} \times Y_{2} \times \ldots \times Y_{n}$ be a fuzzy-anti-n-linear mapping, $X_{1}, X_{2}, \ldots, X_{n}$ and $Y_{1}, Y_{2}, \ldots, Y_{n}$ are subspaces of $\left(X, N_{1}^{*}\right),\left(Y, N_{2}^{*}\right)$ respectively. If $T$ is Fa-n-continuous if and only if $T$ is $S q$-fa- $n$ continuous.

Proof. Let us assume that $T$ is Fa-n-continuous at $\left(x_{0}^{(1)}, x_{0}^{(2)}, x_{0}^{(3)}, \ldots, x_{0}^{(n)}\right) \in X_{1} \times X_{2} \times \ldots \times X_{n}$. Let $\left(x_{k}^{(1)}, x_{k}^{(2)}, x_{k}^{(3)}, \ldots, x_{k}^{(n)}\right)$ be a sequence in $X_{1} \times X_{2} \times \ldots \times X_{n}$, such that $\left(x_{k}^{(1)}, x_{k}^{(2)}, x_{k}^{(3)}, \ldots, x_{k}^{(n)}\right) \rightarrow\left(x_{0}^{(1)}, x_{0}^{(2)}, x_{0}^{(3)}, \ldots, x_{0}^{(n)}\right)$. Let $\varepsilon>0$ be given, choose $\alpha \in(0,1)$, since $T$ is Fa-n-continuous at $\left(x_{0}^{(1)}, x_{0}^{(2)}, x_{0}^{(3)}, \ldots, x_{0}^{(n)}\right)$ then $\exists \delta=\delta(\alpha, \varepsilon)>0, \beta=\beta(\alpha, \varepsilon) \in(0,1)$, such that for all $\left(x^{(1)}, x^{(2)}, x^{(3)}, \ldots, x^{(n)}\right) \in X_{1} \times X_{2} \times \ldots \times X_{n}$,

$$
\begin{aligned}
& N_{1}^{*}\left[\left(x^{(1)}, x^{(2)}, x^{(3)}, \ldots, x^{(n)}\right)-\left(x_{0}^{(1)}, x_{0}^{(2)}, x_{0}^{(3)}, \ldots, x_{0}^{(n)}\right), \delta\right]<\beta \\
\Rightarrow & N_{2}^{*}\left[T\left(x^{(1)}, x^{(2)}, x^{(3)}, \ldots, x^{(n)}\right)-T\left(x_{0}^{(1)}, x_{0}^{(2)}, x_{0}^{(3)}, \ldots, x_{0}^{(n)}\right), \varepsilon\right]<\alpha .
\end{aligned}
$$

Since $\left(x_{k}^{(1)}, x_{k}^{(2)}, x_{k}^{(3)}, \ldots, x_{k}^{(n)}\right) \rightarrow\left(x_{0}^{(1)}, x_{0}^{(2)}, x_{0}^{(3)}, \ldots, x_{0}^{(n)}\right)$ in $\left(X, N_{1}^{*}\right) \exists$ a positive integer $n_{0}$, such that

$$
\begin{aligned}
& N_{1}^{*}\left[\left(x_{k}^{(1)}, x_{k}^{(2)}, x_{k}^{(3)}, \ldots, x_{k}^{(n)}\right)-\left(x_{0}^{(1)}, x_{0}^{(2)}, x_{0}^{(3)}, \ldots, x_{0}^{(n)}\right), \delta\right]<\beta, \forall n \geq n_{0} \\
\Rightarrow & N_{2}^{*}\left[T\left(x_{k}^{(1)}, x_{k}^{(2)}, x_{k}^{(3)}, \ldots, x_{k}^{(n)}\right)-T\left(x_{0}^{(1)}, x_{0}^{(2)}, x_{0}^{(3)}, \ldots, x_{0}^{(n)}\right), \varepsilon\right]<\alpha, \forall n \geq n_{0} \\
\Rightarrow & N_{2}^{*}\left[T\left(x_{k}^{(1)}, x_{k}^{(2)}, x_{k}^{(3)}, \ldots, x_{k}^{(n)}\right)-T\left(x_{0}^{(1)}, x_{0}^{(2)}, x_{0}^{(3)}, \ldots, x_{0}^{(n)}\right), \varepsilon\right]=0 .
\end{aligned}
$$

Since $\varepsilon$ is arbitrary thus $T\left(x_{k}^{(1)}, x_{k}^{(2)}, x_{k}^{(3)}, \ldots, x_{k}^{(n)}\right) \rightarrow T\left(x_{0}^{(1)}, x_{0}^{(2)}, x_{0}^{(3)}, \ldots, x_{0}^{(n)}\right)$ in $Y_{1} \times Y_{2} \times \ldots \times Y_{n}$. Therefore $T$ is Sq-fa-n-continuous.

Next let us assume $T$ is Sq-fa-n-continuous at $\left(x_{0}^{(1)}, x_{0}^{(2)}, x_{0}^{(3)}, \ldots, x_{0}^{(n)}\right) \in X_{1} \times X_{2} \times \ldots \times X_{n}$ If it is possible let us assume $T$ is not Fa-n-continuous at $\left(x_{0}^{(1)}, x_{0}^{(2)}, x_{0}^{(3)}, \ldots, x_{0}^{(n)}\right)$. Thus $\exists \varepsilon>0$ and $\alpha>0$ such that for any $\delta>0$ and $\beta \in(0,1)$ $\exists\left(y^{(1)}, y^{(2)}, y^{(3)}, \ldots, y^{(n)}\right)$ (depending on $\left.\delta, \beta\right)$, such that $N_{1}^{*}\left[\left(x_{0}^{(1)}, x_{0}^{(2)}, x_{0}^{(3)}, \ldots, x_{0}^{(n)}\right)-\left(y^{(1)}, y^{(2)}, y^{(3)}, \ldots, y^{(n)}\right), \delta\right]<\beta$, but $N_{2}^{*}\left[T\left(x_{0}^{(1)}, x_{0}^{(2)}, x_{0}^{(3)}, \ldots, x_{0}^{(n)}\right)-T\left(y^{(1)}, y^{(2)}, y^{(3)}, \ldots, y^{(n)}\right), \varepsilon\right] \geq \alpha$. Thus for $\beta=\frac{1}{k+1}, \delta=1-\frac{1}{k+1}, k=1,2,3, \ldots$, $\exists\left(y_{k}^{(1)}, y_{k}^{(2)}, y_{k}^{(3)}, \ldots, y_{k}^{(n)}\right)$, such that

$$
N_{1}^{*}\left[\left(x_{0}^{(1)}, x_{0}^{(2)}, x_{0}^{(3)}, \ldots, x_{0}^{(n)}\right)-\left(y_{k}^{(1)}, y_{k}^{(2)}, y_{k}^{(3)}, \ldots, y_{k}^{(n)}\right),\left(1-\frac{1}{k+1}\right)\right]<\frac{1}{k+1}
$$

but $N_{2}^{*}\left[T\left(x_{0}^{(1)}, x_{0}^{(2)}, x_{0}^{(3)}, \ldots, x_{0}^{(n)}\right)-T\left(y_{k}^{(1)}, y_{k}^{(2)}, y_{k}^{(3)}, \ldots, y_{k}^{(n)}\right), \varepsilon\right] \geq \alpha$.

Taking $\delta>0, \exists k_{0}$, such that $\left(1-\frac{1}{k+1}\right)<\delta \forall k \geq k_{0}$, then

$$
\begin{gathered}
N_{1}^{*}\left[\left(x_{0}^{(1)}, x_{0}^{(2)}, x_{0}^{(3)}, \ldots, x_{0}^{(n)}\right)-\left(y_{k}^{(1)}, y_{k}^{(2)}, y_{k}^{(3)}, \ldots, y_{k}^{(n)}\right), \delta\right] \\
<N_{1}^{*}\left[\left(x_{0}^{(1)}, x_{0}^{(2)}, x_{0}^{(3)}, \ldots, x_{0}^{(n)}\right)-\left(y_{k}^{(1)}, y_{k}^{(2)}, y_{k}^{(3)}, \ldots, y_{k}^{(n)}\right),\left(1-\frac{1}{k+1}\right)\right]<\frac{1}{k+1}, \forall k>k_{0} \\
\lim _{k \rightarrow \infty} N_{1}^{*}\left[\left(x_{0}^{(1)}, x_{0}^{(2)}, x_{0}^{(3)}, \ldots, x_{0}^{(n)}\right)-\left(y_{k}^{(1)}, y_{k}^{(2)}, y_{k}^{(3)}, \ldots, y_{k}^{(n)}\right), \delta\right]<0 \\
\Rightarrow\left(y_{k}^{(1)}, y_{k}^{(2)}, y_{k}^{(3)}, \ldots, y_{k}^{(n)}\right) \rightarrow\left(x_{0}^{(1)}, x_{0}^{(2)}, x_{0}^{(3)}, \ldots, x_{0}^{(n)}\right) .
\end{gathered}
$$

But from Equation (1) $N_{2}^{*}\left[T\left(x_{0}^{(1)}, x_{0}^{(2)}, x_{0}^{(3)}, \ldots, x_{0}^{(n)}\right)-T\left(y_{k}^{(1)}, y_{k}^{(2)}, y_{k}^{(3)}, \ldots, y_{k}^{(n)}\right), \varepsilon\right] \geq \alpha$. So, $N_{2}^{*}\left[T\left(x_{0}^{(1)}, x_{0}^{(2)}, x_{0}^{(3)}, \ldots, x_{0}^{(n)}\right)-\right.$ $\left.T\left(y_{k}^{(1)}, y_{k}^{(2)}, y_{k}^{(3)}, \ldots, y_{k}^{(n)}\right), \varepsilon\right]$ does not converges to zero as $\mathrm{k} \rightarrow \infty$. Thus $T\left(y_{k}^{(1)}, y_{k}^{(2)}, y_{k}^{(3)}, \ldots, y_{k}^{(n)}\right)$ does not converges to $T\left(x_{0}^{(1)}, x_{0}^{(2)}, x_{0}^{(3)}, \ldots, x_{0}^{(n)}\right)$, where as $\left(y_{k}^{(1)}, y_{k}^{(2)}, y_{k}^{(3)}, \ldots, y_{k}^{(n)}\right) \rightarrow\left(x_{0}^{(1)}, x_{0}^{(2)}, x_{0}^{(3)}, \ldots, x_{0}^{(n)}\right)$ (with respect to $\left.N_{1}^{*}\right)$. This would be contradiction to above assumption. Therefore $T$ is Fa-n-continuous at $\left(x_{0}^{(1)}, x_{0}^{(2)}, x_{0}^{(3)}, \ldots, x_{0}^{(n)}\right)$. 


\section{Fuzzy Anti n-Bounded Linear Operators}

Definition 4.1 Let $T: X_{1} \times X_{2} \times \ldots \times X_{n} \rightarrow Y_{1} \times Y_{2} \times \ldots \times Y_{n}$ be a fuzzy-anti-n-linear mapping, $X_{1}, X_{2}, \ldots, X_{n}$ and $Y_{1}, Y_{2}, \ldots, Y_{n}$ are subspaces of $\left(X, N_{1}^{*}\right),\left(Y, N_{2}^{*}\right)$ respectively. Then $T$ is said to be strongly-fuzzy-anti-n-bounded (St-fa-n-bounded) on $X_{1} \times X_{2} \times \ldots \times X_{n}$ if and only if $\exists$ a positive real number $M$, such that for all $\left(x_{1}, x_{2}, x_{3}, \ldots, x_{n}\right) \in$ $X_{1} \times X_{2} \times \ldots \times X_{n}$ and $\forall t \in R$,

$$
N_{2}^{*}\left[T\left(x_{1}, x_{2}, x_{3}, \ldots, x_{n}\right), t\right] \leq N_{1}^{*}\left[\left(x_{1}, x_{2}, x_{3}, \ldots, x_{n}\right), \frac{t}{M}\right]
$$

Example 4.2 Let $(X,\|\bullet, \bullet, \ldots, \bullet\|)$ be a n-normed-linear-space over the field $K$, where $K=R$ or $C$. Let $k_{1}, k_{2} \in R$ such that $k_{1}>k_{2}>0$. Let $N_{1}^{*}, N_{2}^{*}: X \times X \times \ldots \times X \times R^{+} \rightarrow[0,1]$ be defined by

$$
\begin{aligned}
& N_{1}^{*}\left[\left(x_{1}, x_{2}, x_{3}, \ldots, x_{n}, t\right)\right]=\frac{k_{1}\left\|x_{1}, x_{2}, x_{3}, \ldots, x_{n}\right\|}{t+k_{1}\left\|x_{1}, x_{2}, x_{3}, \ldots, x_{n}\right\|}, \\
& N_{2}^{*}\left[\left(x_{1}, x_{2}, x_{3}, \ldots, x_{n}, t\right)\right]=\frac{k_{2}\left\|x_{1}, x_{2}, x_{3}, \ldots, x_{n}\right\|}{t+k_{2}\left\|x_{1}, x_{2}, x_{3}, \ldots, x_{n}\right\|} .
\end{aligned}
$$

Clearly $\left(X, N_{1}^{*}\right)$ and $\left(Y, N_{2}^{*}\right)$ are fuzzy-anti-n-normed linear spaces.

Consider the mapping $T: X_{1} \times X_{2} \times \ldots \times X_{n} \rightarrow Y_{1} \times Y_{2} \times \ldots \times Y_{n}$ defined by $T\left(x_{1}, x_{2}, x_{3}, \ldots, x_{n}\right)=r\left(x_{1}, x_{2}, x_{3}, \ldots, x_{n}\right)$, where $r(\neq 0) \in R$ is fixed.

Clearly $T$ is a linear operator. Let us choose an arbitrary but fixed $M>0$ such that $M \geq|r|$ and $\left(x_{1}, x_{2}, x_{3}, \ldots, x_{n}\right) \in$ $X_{1} \times X_{2} \times \ldots \times X_{n}$. Now

$$
\begin{gathered}
M \geq|r| \\
\Rightarrow \quad k_{1} M\left\|x_{1}, x_{2}, x_{3}, \ldots, x_{n}\right\| \geq k_{2}|r|\left\|x_{1}, x_{2}, x_{3}, \ldots, x_{n}\right\| \\
\Rightarrow \quad t+k_{1} M\left\|x_{1}, x_{2}, x_{3}, \ldots, x_{n}\right\| \geq t+k_{2}|r|\left\|x_{1}, x_{2}, x_{3}, \ldots, x_{n}\right\|, \forall t>0 \\
\Rightarrow \quad \frac{t}{t+k_{2}|r|\left\|x_{1}, x_{2}, x_{3}, \ldots, x_{n}\right\|} \geq \frac{t}{t+k_{1} M\left\|x_{1}, x_{2}, x_{3}, \ldots, x_{n}\right\|}, \forall t>0 \\
\Rightarrow \quad \frac{t}{t+k_{2}\left\|r\left(x_{1}, x_{2}, x_{3}, \ldots, x_{n}\right)\right\|} \geq \frac{\frac{t}{M}}{\frac{t}{M}+k_{1}\left\|x_{1}, x_{2}, x_{3}, \ldots, x_{n}\right\|}, \forall t>0 \\
\Rightarrow \quad 1-\frac{t}{t+k_{2}\left\|r\left(x_{1}, x_{2}, x_{3}, \ldots, x_{n}\right)\right\|} \leq 1-\frac{\frac{t}{M}}{\frac{t}{M}+k_{1}\left\|x_{1}, x_{2}, x_{3}, \ldots, x_{n}\right\|}, \forall t>0 \\
\Rightarrow \quad \frac{k_{2}\left\|r\left(x_{1}, x_{2}, x_{3}, \ldots, x_{n}\right)\right\|}{t+k_{2}\left\|r\left(x_{1}, x_{2}, x_{3}, \ldots, x_{n}\right)\right\|} \leq \frac{k_{1}\left\|x_{1}, x_{2}, x_{3}, \ldots, x_{n}\right\|}{\frac{t}{M}+k_{1}\left\|x_{1}, x_{2}, x_{3}, \ldots, x_{n}\right\|}, \forall t>0 . \\
\quad N_{2}^{*}\left[r\left(x_{1}, x_{2}, x_{3}, \ldots, x_{n}\right), t\right] \leq N_{1}^{*}\left[\left(x_{1}, x_{2}, x_{3}, \ldots, x_{n}\right), \frac{t}{M}\right], \forall t>0
\end{gathered}
$$

and

$$
\left(x_{1}, x_{2}, x_{3}, \ldots, x_{n}\right) \in X_{1} \times X_{2} \times \ldots \times X_{n}
$$

(i.e.)

$$
N_{2}^{*}\left[T\left(x_{1}, x_{2}, x_{3}, \ldots, x_{n}\right), t\right] \leq N_{1}^{*}\left[\left(x_{1}, x_{2}, x_{3}, \ldots, x_{n}\right), \frac{t}{M}\right], \forall t>0
$$

and

$$
\left(x_{1}, x_{2}, x_{3}, \ldots, x_{n}\right) \in X_{1} \times X_{2} \times \ldots \times X_{n} .
$$

Therefore $T$ is St-fa-n-bounded.

Definition 4.3 Let $T: X_{1} \times X_{2} \times \ldots \times X_{n} \rightarrow Y_{1} \times Y_{2} \times \ldots \times Y_{n}$ be a fuzzy-anti-n-linear mapping, $X_{1}, X_{2}, \ldots, X_{n}$ and $Y_{1}, Y_{2}, \ldots, Y_{n}$ are subspaces of $\left(X, N_{1}^{*}\right),\left(Y, N_{2}^{*}\right)$ respectively. Then $T$ is said to be weakly-fuzzy-anti-n-bounded (Wkfa-n-bounded) on $X_{1} \times X_{2} \times \ldots \times X_{n}$ iff for any $\alpha \in(0,1) \exists M_{\alpha}>0$, such that for all $\left(x_{1}, x_{2}, x_{3}, \ldots, x_{n}\right) \in X_{1} \times X_{2} \times \ldots \times X_{n}$ and $\forall t \in R$,

$$
N_{1}^{*}\left[\left(x_{1}, x_{2}, x_{3}, \ldots, x_{n}\right), \frac{t}{M}\right] \leq 1-\alpha \Rightarrow N_{2}^{*}\left[T\left(x_{1}, x_{2}, x_{3}, \ldots, x_{n}\right), t\right] \leq 1-\alpha
$$


Theorem 4.4 Let T: $X_{1} \times X_{2} \times \ldots \times X_{n} \rightarrow Y_{1} \times Y_{2} \times \ldots \times Y_{n}$ be a fuzzy-anti-n-linear operator, $X_{1}, X_{2}, \ldots, X_{n}$ and $Y_{1}, Y_{2}, \ldots, Y_{n}$ are subspaces of $\left(X, N_{1}^{*}\right),\left(Y, N_{2}^{*}\right)$ respectively. If $T$ is $S t-f a-n$-bounded, then $T$ is Wk-fa-n-bounded but the converse need not be sure.

Proof. Let us assume $T$ is St-fa-n-bounded. Then $\exists M>0$, such that $\forall\left(x_{1}, x_{2}, x_{3}, \ldots, x_{n}\right) \in X_{1} \times X_{2} \times \ldots \times X_{n}$ and $\forall t \in R, N_{2}^{*}\left[T\left(x_{1}, x_{2}, x_{3}, \ldots, x_{n}\right), t\right] \leq N_{1}^{*}\left[\left(x_{1}, x_{2}, x_{3}, \ldots, x_{n}\right), \frac{t}{M}\right]$. Thus for any $\alpha \in(0,1), \exists M_{\alpha}(=M)>0$, such that

$$
N_{1}^{*}\left[\left(x_{1}, x_{2}, x_{3}, \ldots, x_{n}\right), \frac{t}{M_{\alpha}}\right] \leq 1-\alpha \Rightarrow N_{2}^{*}\left[T\left(x_{1}, x_{2}, x_{3}, \ldots, x_{n}\right), t\right] \leq 1-\alpha .
$$

Therefore $T$ is Wk-fa-n-bounded.

The following example tells us that the converse of the theorem is not always true.

Example 4.5 Let $(X,\|\bullet, \bullet, \ldots, \bullet\|)$ be a n-normed-linear space over the field $K$, where $K=R$ or $C$. Let $N_{1}^{*}, N_{2}^{*}$ : $X \times X \times \ldots \times X \times R^{+} \rightarrow[0,1]$ be defined by $N_{1}^{*}\left(x_{1}, x_{2}, x_{3}, \ldots, x_{n}, t\right)=\frac{4\left\|x_{1}, x_{2}, x_{3}, \ldots, x_{n}\right\|^{2}}{t^{2}+2\left\|x_{1}, x_{2}, x_{3}, \ldots, x_{n}\right\|^{2}}$ if $t>\left\|x_{1}, x_{2}, x_{3}, \ldots, x_{n}\right\|=1$, if $t \leq\left\|x_{1}, x_{2}, x_{3}, \ldots, x_{n}\right\|$

$$
N_{2}^{*}\left(x_{1}, x_{2}, x_{3}, \ldots, x_{n}, t\right)=\frac{\left\|x_{1}, x_{2}, x_{3}, \ldots, x_{n}\right\|}{t+\left\|x_{1}, x_{2}, x_{3}, \ldots, x_{n}\right\|} .
$$

We know that $\left(X, N_{2}^{*}\right)$ is a Fa-n-normed linear space.

Now we would prove $\left(X, N_{1}^{*}\right)$ is a Fa-n-normed linear space.

(i) $\forall t \in R$ with $t \leq 0$ and by definition $N_{1}^{*}\left(x_{1}, x_{2}, x_{3}, \ldots, x_{n}, t\right)=1$

(ii) $\forall t \in R$ with $t>0$,

$$
\begin{gathered}
N_{1}^{*}\left(x_{1}, x_{2}, x_{3}, \ldots, x_{n}, t\right)=0 \Leftrightarrow \frac{4\left\|x_{1}, x_{2}, x_{3}, \ldots, x_{n}\right\|^{2}}{t^{2}+2\left\|x_{1}, x_{2}, x_{3}, \ldots, x_{n}\right\|^{2}}=0 \\
\Leftrightarrow\left\|x_{1}, x_{2}, x_{3}, \ldots, x_{n}\right\|^{2}=0 \Leftrightarrow x_{1}, x_{2}, x_{3}, \ldots, x_{n} \text { are linearly dependent. }
\end{gathered}
$$

(iii) As $\left\|x_{1}, x_{2}, x_{3}, \ldots, x_{n}\right\|$ is invariant under any permutation of $x_{1}, x_{2}, x_{3}, \ldots, x_{n}$ it follows that $N_{1}^{*}\left(x_{1}, x_{2}, x_{3}, \ldots, x_{n}, t\right)$ is invariant under any permutation of $x_{1}, x_{2}, x_{3}, \ldots, x_{n}$.

(iv) For all $t \in R$ with $t>0$ and $c \neq 0, c \in K$, we get

$$
\begin{gathered}
N_{1}^{*}\left(x_{1}, x_{2}, x_{3}, \ldots, c x_{n}, t\right)=\frac{4\left\|x_{1}, x_{2}, x_{3}, \ldots, c x_{n}\right\|^{2}}{t^{2}+2\left\|x_{1}, x_{2}, x_{3}, \ldots, c x_{n}\right\|^{2}}=\frac{|c|^{2} 4\left\|x_{1}, x_{2}, x_{3}, \ldots, x_{n}\right\|^{2}}{t^{2}+|c|^{2} 2\left\|x_{1}, x_{2}, x_{3}, \ldots, x_{n}\right\|^{2}} \\
=\frac{4\left\|x_{1}, x_{2}, x_{3}, \ldots, x_{n}\right\|^{2}}{\frac{t^{2}}{|c|^{2}}+2\left\|x_{1}, x_{2}, x_{3}, \ldots, x_{n}\right\|^{2}}=N_{1}^{*}\left[\left(x_{1}, x_{2}, x_{3}, \ldots, x_{n}, \frac{t}{|c|}\right)\right] .
\end{gathered}
$$

(v) For all $s, t \in R$ and $x_{1}, x_{2}, x_{3}, \ldots, x_{n}, x_{n}^{\prime} \in X$, we have to show that

$$
N_{1}^{*}\left(x_{1}, x_{2}, \ldots, x_{n-1}, x_{n}+x_{n}^{\prime}, s+t\right) \leq \max \left\{N_{1}^{*}\left(x_{1}, x_{2}, \ldots, x_{n-1}, x_{n}, s\right), N_{1}^{*}\left(x_{1}, x_{2}, \ldots, x_{n-1}, x_{n}^{\prime}, t\right)\right\} .
$$

If (a) $s+t<0$ (b) $s=t=0$ (c) $s+t>0, s>0, t<0$; $s<0, t>0$, then in the three cases the relation will be trivial.

If (d) $s>0, t>0, s+t>0$ and

$$
\left\|x_{1}, x_{2}, x_{3}, \ldots ., x_{n-1}, x_{n}\right\|+\left\|x_{1}, x_{2}, x_{3}, \ldots ., x_{n-1} x_{n}^{\prime}\right\| \geq\left\|x_{1}, x_{2}, x_{3}, \ldots ., x_{n-1}, x_{n}+x_{n}^{\prime}\right\| .
$$

Therefore

$$
\begin{gathered}
N_{1}^{*}\left(x_{1}, x_{2}, x_{3}, \ldots, x_{n-1}, x_{n}+x_{n}^{\prime}, s+t\right)=\frac{4\left\|x_{1}, x_{2}, x_{3}, \ldots, x_{n-1},\left(x_{n}+x_{n}^{\prime}\right)\right\|^{2}}{(s+t)^{2}+2\left\|x_{1}, x_{2}, x_{3}, \ldots, x_{n-1},\left(x_{n}+x_{n}^{\prime}\right)\right\|^{2}} \\
\leq \frac{4\left(\left\|x_{1}, x_{2}, x_{3}, \ldots, x_{n}\right\|+\left\|x_{1}, x_{2}, x_{3}, \ldots, x_{n-1}, x_{n}^{\prime}\right\|\right)^{2}}{(s+t)^{2}+2\left(\left\|x_{1}, x_{2}, x_{3}, \ldots, x_{n-1}, x_{n}\right\|+\left\|x_{1}, x_{2}, x_{3}, \ldots, x_{n-1}, x_{n}^{\prime}\right\|\right)^{2}} \\
\leq \frac{4\left\|x_{1}, x_{2}, x_{3}, \ldots, x_{n-1}, x_{n}^{\prime}\right\|^{2}}{t^{2}+2\left\|x_{1}, x_{2}, x_{3}, \ldots, x_{n-1}, x_{n}^{\prime}\right\|^{2}}=N_{1}^{*}\left(x_{1}, x_{2}, x_{3}, \ldots, x_{n-1}, x_{n}^{\prime}, t\right) .
\end{gathered}
$$


Therefore $N_{1}^{*}\left(x_{1}, x_{2}, x_{3}, \ldots, x_{n-1}, x_{n}+x_{n}^{\prime}, s+t\right) \leq N_{1}^{*}\left(x_{1}, x_{2}, x_{3}, \ldots, x_{n-1}, x_{n}^{\prime}, t\right)$, when $N_{1}^{*}\left(x_{1}, x_{2}, x_{3}, \ldots, x_{n-1}, x_{n}, s\right) \leq$ $N_{1}^{*}\left(x_{1}, x_{2}, x_{3}, \ldots ., x_{n-1}, x_{n}, t\right)$. Similarly, $N_{1}^{*}\left(x_{1}, x_{2}, x_{3}, \ldots, x_{n-1}, x_{n}+x_{n}^{\prime}, s+t\right) \leq N_{1}^{*}\left(x_{1}, x_{2}, x_{3}, \ldots, x_{n-1}, x_{n}, s\right)$, when $N_{1}^{*}\left(x_{1}, x_{2}, x_{3}, \ldots, x_{n-1}, x_{n}^{\prime}, t\right) \leq N_{1}^{*}\left(x_{1}, x_{2}, x_{3}, \ldots, x_{n-1}, x_{n}, s\right)$. Thus $N_{1}^{*}\left(x_{1}, x_{2}, \ldots, x_{n-1}, x_{n}+x_{n}^{\prime}, s+t\right) \leq \max \left\{N_{1}^{*}\left(x_{1}, x_{2}\right.\right.$, $\left.\left.\ldots, x_{n-1}, x_{n}, s\right), N_{1}^{*}\left(x_{1}, x_{2}, \ldots, x_{n-1}, x_{n}^{\prime}, t\right)\right\}$.

If $t_{1}<t_{2} \leq 0$, which implies

$$
N_{1}^{*}\left(x_{1}, x_{2}, \ldots, x_{n-1}, x_{n}, t_{1}\right)=N_{1}^{*}\left(x_{1}, x_{2}, \ldots, x_{n-1}, x_{n}, t_{2}\right)=1 .
$$

If $0<t_{1}<t_{2}$, then

$$
\begin{aligned}
& N_{1}^{*}\left(x_{1}, x_{2}, \ldots ., x_{n}, t_{1}\right)-N_{1}^{*}\left(x_{1}, x_{2}, \ldots ., x_{n}, t_{2}\right) \\
= & \frac{4\left\|x_{1}, x_{2}, \ldots ., x_{n}\right\|^{2}}{t_{1}^{2}+2\left\|x_{1}, x_{2}, \ldots ., x_{n}\right\|^{2}}-\frac{4\left\|x_{1}, x_{2}, \ldots ., x_{n}\right\|^{2}}{t_{2}^{2}+2\left\|x_{1}, x_{2}, \ldots ., x_{n}\right\|^{2}} \\
= & \frac{4\left\|x_{1}, x_{2}, \ldots ., x_{n}\right\|^{2}\left(t_{2}^{2}-t_{1}^{2}\right)}{\left(t_{1}^{2}+2\left\|x_{1}, x_{2}, \ldots ., x_{n}\right\|^{2}\right)\left(t_{2}^{2}+2\left\|x_{1}, x_{2}, \ldots ., x_{n}\right\|^{2}\right)}>0 \\
\Rightarrow & N_{1}^{*}\left(x_{1}, x_{2}, x_{3}, \ldots, x_{n}, t_{1}\right) \geq N_{1}^{*}\left(x_{1}, x_{2}, x_{3}, \ldots ., x_{n}, t_{2}\right) .
\end{aligned}
$$

Thus $N_{1}^{*}\left(x_{1}, x_{2}, x_{3}, \ldots, x_{n}, t\right)$ is a non- increasing function of $t \in R$

$$
\lim _{t \rightarrow \infty} N_{1}^{*}\left(x_{1}, x_{2}, x_{3}, \ldots, x_{n}, t\right)=\lim _{t \rightarrow \infty} \frac{4\left\|x_{1}, x_{2}, x_{3}, \ldots, x_{n}\right\|^{2}}{t^{2}+2\left\|x_{1}, x_{2}, x_{3}, \ldots, x_{n}\right\|^{2}}=0, \forall\left(x_{1}, x_{2}, x_{3}, \ldots, x_{n}\right) \in X_{1} \times X_{2} \times \ldots \times X_{n} .
$$

Therefore $\left(X, N_{1}^{*}\right)$ is a fuzzy-anti-n-normed linear space.

Now let us consider the mapping $T: X_{1} \times X_{2} \times \ldots \times X_{n} \rightarrow Y_{1} \times Y_{2} \times \ldots \times Y_{n}$ defined by

$$
T\left(x_{1}, x_{2}, x_{3}, \ldots, x_{n}\right)=\left(x_{1}, x_{2}, x_{3}, \ldots, x_{n}\right) \forall\left(x_{1}, x_{2}, x_{3}, \ldots, x_{n}\right) \in X_{1} \times X_{2} \times X_{3} \times \ldots . . \times X_{n}
$$

Let $\alpha \in(0,1)$ and $t \in R^{+}$and choose $M_{\alpha}=\frac{1}{1-\alpha}$.

We now prove that

$$
\begin{gathered}
N_{1}^{*}\left[\left(x_{1}, x_{2}, x_{3}, \ldots, x_{n}\right), \frac{t}{M_{\alpha}}\right] \leq 1-\alpha \Rightarrow N_{2}^{*}\left[T\left(x_{1}, x_{2}, x_{3}, \ldots, x_{n}\right), t\right] \leq 1-\alpha \\
N_{1}^{*}\left[\left(x_{1}, x_{2}, x_{3}, \ldots, x_{n}\right), \frac{t}{M_{\alpha}}\right] \leq 1-\alpha \Rightarrow \frac{4\left\|x_{1}, x_{2}, x_{3}, \ldots, x_{n}\right\|^{2}}{t^{2}(1-\alpha)^{2}+2\left\|x_{1}, x_{2}, x_{3}, \ldots, x_{n}\right\|^{2}} \leq 1-\alpha \\
\Rightarrow 1-\frac{4\left\|x_{1}, x_{2}, x_{3}, \ldots, x_{n}\right\|^{2}}{t^{2}(1-\alpha)^{2}+2\left\|x_{1}, x_{2}, x_{3}, \ldots, x_{n}\right\|^{2}} \geq 1-(1-\alpha)=\alpha \\
\Rightarrow \frac{t^{2}(1-\alpha)^{2}-2\left\|x_{1}, x_{2}, x_{3}, \ldots, x_{n}\right\|^{2}}{t^{2}(1-\alpha)^{2}+2\left\|x_{1}, x_{2}, x_{3}, \ldots, x_{n}\right\|^{2}} \geq \alpha \\
\Rightarrow t^{2}(1-\alpha)^{2}-2\left\|x_{1}, x_{2}, x_{3}, \ldots, x_{n}\right\|^{2} \geq t^{2} \alpha(1-\alpha)^{2}+2 \alpha\left\|x_{1}, x_{2}, x_{3}, \ldots, x_{n}\right\|^{2} \\
\Rightarrow t^{2}(1-\alpha)^{3} \geq 2(1+\alpha)\left\|x_{1}, x_{2}, x_{3}, \ldots, x_{n}\right\|^{2} \\
\Rightarrow\left\|x_{1}, x_{2}, x_{3}, \ldots, x_{n}\right\|^{2} \leq \frac{t^{2}(1-\alpha)^{3}}{2(1+\alpha)} \\
\Rightarrow\left\|x_{1}, x_{2}, x_{3}, \ldots, x_{n}\right\| \leq \frac{t(1-\alpha) \sqrt{(1-\alpha)}}{\sqrt{2} \sqrt{(1+\alpha)}} \\
\Rightarrow t+\left\|x_{1}, x_{2}, x_{3}, \ldots, x_{n}\right\| \leq \frac{t \sqrt{2} \sqrt{(1+\alpha)}+t(1-\alpha) \sqrt{(1-\alpha)}}{\sqrt{2} \sqrt{(1+\alpha)}} \\
t \\
\Rightarrow \frac{\sqrt{2} \sqrt{(1+\alpha)}}{t+\left\|x_{1}, x_{2}, x_{3}, \ldots, x_{n}\right\|} \geq \frac{(1-\alpha) \sqrt{(1-\alpha)}+\sqrt{2} \sqrt{(1+\alpha)}}{77}
\end{gathered}
$$




$$
\begin{gathered}
\Rightarrow 1-\frac{t}{t+\left\|x_{1}, x_{2}, x_{3}, \ldots, x_{n}\right\|} \leq 1-\frac{\sqrt{2} \sqrt{(1+\alpha)}}{(1-\alpha) \sqrt{(1-\alpha)}+\sqrt{2} \sqrt{(1+\alpha)}} \\
\Rightarrow \frac{\left\|x_{1}, x_{2}, x_{3}, \ldots, x_{n}\right\|}{t+\left\|x_{1}, x_{2}, x_{3}, \ldots, x_{n}\right\|} \leq \frac{(1-\alpha) \sqrt{(1-\alpha)}}{(1-\alpha) \sqrt{(1-\alpha)}+\sqrt{2} \sqrt{(1+\alpha)}}
\end{gathered}
$$

Now consider

$$
\begin{gathered}
\frac{(1-\alpha) \sqrt{(1-\alpha)}}{(1-\alpha) \sqrt{(1-\alpha)}+\sqrt{2} \sqrt{(1+\alpha)}} \leq(1-\alpha) \\
\Leftrightarrow \sqrt{(1-\alpha)} \leq \sqrt{2} \sqrt{(1+\alpha)}+\sqrt{(1-\alpha)}-\alpha \sqrt{(1-\alpha)} \\
\Leftrightarrow 0 \leq \sqrt{2} \sqrt{(1+\alpha)}-\alpha \sqrt{(1-\alpha)} \Leftrightarrow \alpha \sqrt{(1-\alpha)} \leq \sqrt{2} \sqrt{(1+\alpha)} \\
\Leftrightarrow \alpha^{2}(1-\alpha) \leq 2+2 \alpha \Leftrightarrow \alpha^{2} \leq \alpha^{3}+2 \alpha+2,
\end{gathered}
$$

which is true for all $\alpha \in(0,1)$.

Hence

$$
N_{1}^{*}\left[\left(x_{1}, x_{2}, x_{3}, \ldots, x_{n}\right), \frac{t}{M_{\alpha}}\right] \leq 1-\alpha \Rightarrow N_{2}^{*}\left[T\left(x_{1}, x_{2}, x_{3}, \ldots, x_{n}\right), t\right] \leq 1-\alpha .
$$

Therefore $T$ is weakly-fuzzy-anti-n-bounded.

Now conversely, let $T$ be St-fa-n-bounded.

$$
\begin{gathered}
N_{2}^{*}\left[T\left(x_{1}, x_{2}, x_{3}, \ldots, x_{n}\right), t\right] \leq N_{1}^{*}\left[\left(x_{1}, x_{2}, x_{3}, \ldots, x_{n}\right), \frac{t}{M_{\alpha}}\right] \\
\frac{\left\|x_{1}, x_{2}, x_{3}, \ldots, x_{n}\right\|}{t+\left\|x_{1}, x_{2}, x_{3}, \ldots, x_{n}\right\|} \leq \frac{4\left\|x_{1}, x_{2}, x_{3}, \ldots, x_{n}\right\|^{2}}{\frac{t^{2}}{M^{2}}+2\left\|x_{1}, x_{2}, x_{3}, \ldots, x_{n}\right\|^{2}}\left\{M_{\alpha}=M\right\} \\
\frac{\left\|x_{1}, x_{2}, x_{3}, \ldots, x_{n}\right\|}{t+\left\|x_{1}, x_{2}, x_{3}, \ldots, x_{n}\right\|} \leq \frac{4 M^{2}\left\|x_{1}, x_{2}, x_{3}, \ldots, x_{n}\right\|^{2}}{t^{2}+2 M^{2}\left\|x_{1}, x_{2}, x_{3}, \ldots, x_{n}\right\|^{2}} \\
\Leftrightarrow t^{2}\left\|x_{1}, x_{2}, x_{3}, \ldots, x_{n}\right\|+2 M^{2}\left\|x_{1}, x_{2}, x_{3}, \ldots, x_{n}\right\|^{3} \leq 4 t M^{2}\left\|x_{1}, x_{2}, x_{3}, \ldots, x_{n}\right\|^{2}+4 M^{2}\left\|x_{1}, x_{2}, x_{3}, \ldots, x_{n}\right\|^{3} \\
\Leftrightarrow t^{2}\left\|x_{1}, x_{2}, x_{3}, \ldots, x_{n}\right\| \leq 4 t M^{2}\left\|x_{1}, x_{2}, x_{3}, \ldots, x_{n}\right\|^{2}+2 M^{2}\left\|x_{1}, x_{2}, x_{3}, \ldots, x_{n}\right\|^{3} \\
\Leftrightarrow t^{2} \leq 4 t M^{2}\left\|x_{1}, x_{2}, x_{3}, \ldots, x_{n}\right\|+2 M^{2}\left\|x_{1}, x_{2}, x_{3}, \ldots, x_{n}\right\|^{2} \\
\Leftrightarrow \frac{t^{2}}{4 t\left\|x_{1}, x_{2}, x_{3}, \ldots, x_{n}\right\|+2\left\|x_{1}, x_{2}, x_{3}, \ldots, x_{n}\right\|^{2}} \leq M^{2},
\end{gathered}
$$

(i.e.)

$$
\begin{aligned}
M^{2} & \geq \frac{t^{2}}{4 t\left\|x_{1}, x_{2}, x_{3}, \ldots, x_{n}\right\|+2\left\|x_{1}, x_{2}, x_{3}, \ldots, x_{n}\right\|^{2}}, \text { for } t \in(0,1) \\
& \Leftrightarrow M \geq \frac{t}{\left(4 t\left\|x_{1}, x_{2}, x_{3}, \ldots, x_{n}\right\|+2\left\|x_{1}, x_{2}, x_{3}, \ldots, x_{n}\right\|^{2}\right)^{\frac{1}{2}}} .
\end{aligned}
$$

$M=\infty$ as $t \rightarrow \infty$. This would be contradiction to above assumption. Therefore $T$ is not St-fa-n-bounded.

Theorem 4.6 Let $T: X_{1} \times X_{2} \times \ldots \times X_{n} \rightarrow Y_{1} \times Y_{2} \times \ldots \times Y_{n}$ be a fuzzy-anti-n-linear mapping, $X_{1}, X_{2}, \ldots, X_{n}$ and $Y_{1}, Y_{2}, \ldots, Y_{n}$ are subspaces of $\left(X, N_{1}^{*}\right),\left(Y, N_{2}^{*}\right)$ respectively. Then

(i) $T$ is St-fa-n-continuous on $X_{1} \times X_{2} \times \ldots \times X_{n}$, if T is St-fa-n-continuous at a point $\left(x_{0}^{(1)}, x_{0}^{(2)}, \ldots ., x_{0}^{(n)}\right) \in X_{1} \times X_{2} \times$ $\ldots \times X_{n}$;

(ii) $T$ is St-fa-n-continuous iff $T$ is St-fa-n-bounded.

Proof. (i) Since $T$ is St-fa-n-continuous at $\left(x_{0}^{(1)}, x_{0}^{(2)}, \ldots \ldots, x_{0}^{(n)}\right) \in X_{1} \times X_{2} \times \ldots \times X_{n}$, if for each $\varepsilon>0$, there exists $\delta>0$, such that

$$
N_{2}^{*}\left[T\left(x^{(1)}, x^{(2)}, \ldots, x^{(n)}\right)-T\left(x_{0}^{(1)}, x_{0}^{(2)}, \ldots, x_{0}^{(n)}\right), \varepsilon\right] \leq N_{1}^{*}\left[\left(x^{(1)}, x^{(2)}, \ldots, x^{(n)}\right)-\left(x_{0}^{(1)}, x_{0}^{(2)}, \ldots, x_{0}^{(n)}\right), \delta\right],
$$


taking $\left(y^{(1)}, y^{(2)}, \ldots . ., y^{(n)}\right) \in X_{1} \times X_{2} \times \ldots \times X_{n}$ and replacing $\left(x^{(1)}, x^{(2)}, \ldots . ., x^{(n)}\right)$ by $\left(x^{(1)}, x^{(2)}, \ldots, x^{(n)}\right)+\left(x_{0}^{(1)}, x_{0}^{(2)}, \ldots, x_{0}^{(n)}\right)-$ $\left(y^{(1)}, y^{(2)}, \ldots, y^{(n)}\right)$, we get

$$
\begin{gathered}
N_{2}^{*}\left[T\left[\left(x^{(1)}, x^{(2)}, \ldots, x^{(n)}\right)+\left(x_{0}^{(1)}, x_{0}^{(2)}, \ldots, x_{0}^{(n)}\right)-\left(y^{(1)}, y^{(2)}, \ldots, y^{(n)}\right)\right]-T\left(x_{0}^{(1)}, x_{0}^{(2)}, \ldots, x_{0}^{(n)}\right), \varepsilon\right] \\
\leq N_{1}^{*}\left[\left(x^{(1)}, x^{(2)}, \ldots, x^{(n)}\right)+\left(x_{0}^{(1)}, x_{0}^{(2)}, \ldots, x_{0}^{(n)}\right)-\left(y^{(1)}, y^{(2)}, \ldots, y^{(n)}\right)-\left(x_{0}^{(1)}, x_{0}^{(2)}, \ldots, x_{0}^{(n)}\right), \delta\right] \\
\Rightarrow N_{2}^{*}\left[T\left(x^{(1)}, x^{(2)}, \ldots, x^{(n)}\right)-T\left(y^{(1)}, y^{(2)}, \ldots, y^{(n)}\right), \varepsilon\right] \leq N_{1}^{*}\left[\left(x^{(1)}, x^{(2)}, \ldots, x^{(n)}\right)-\left(y^{(1)}, y^{(2)}, \ldots, y^{(n)}\right), \delta\right] .
\end{gathered}
$$

Since $\left(y^{(1)}, y^{(2)}, \ldots \ldots, y^{(n)}\right) \in X_{1} \times X_{2} \times \ldots \times X_{n}$ is arbitrary. Therefore $T$ is St-fa-n-continuous on $X_{1} \times X_{2} \times \ldots \times X_{n}$.

(ii) Now we assume $T$ is St-fa-n-bounded. Thus there exists a positive real number $M$, such that for all $\left(x^{(1)}, x^{(2)}, \ldots\right.$, $\left.x^{(n)}\right) \in X_{1} \times X_{2} \times \ldots \times X_{n}$ and $\forall \varepsilon \in R^{+}$,

$$
\begin{gathered}
N_{2}^{*}\left[T\left(x^{(1)}, x^{(2)}, \ldots, x^{(n)}\right), \varepsilon\right] \leq N_{1}^{*}\left[\left(x^{(1)}, x^{(2)}, \ldots, x^{(n)}\right), \frac{\varepsilon}{M}\right] \\
N_{2}^{*}\left[T\left(x^{(1)}, x^{(2)}, \ldots, x^{(n)}\right)-T\left(x_{0}^{(1)}, x_{0}^{(2)}, \ldots, x_{0}^{(n)}\right), \varepsilon\right] \leq N_{1}^{*}\left[\left(x^{(1)}, x^{(2)}, \ldots, x^{(n)}\right)-\left(x_{0}^{(1)}, x_{0}^{(2)}, \ldots, x_{0}^{(n)}\right), \frac{\varepsilon}{M}\right] \\
\Rightarrow N_{2}^{*}\left[T\left(x^{(1)}, x^{(2)}, \ldots, x^{(n)}\right)-T\left(x_{0}^{(1)}, x_{0}^{(2)}, \ldots, x_{0}^{(n)}\right), \varepsilon\right] \leq N_{1}^{*}\left[\left(x^{(1)}, x^{(2)}, \ldots, x^{(n)}\right)-\left(x_{0}^{(1)}, x_{0}^{(2)}, \ldots, x_{0}^{(n)}\right), \delta\right]
\end{gathered}
$$

where $\delta=\frac{\varepsilon}{M}$. Therefore $T$ is St-fa-n-continuous at $\left(x_{0}^{(1)}, x_{0}^{(2)}, \ldots, x_{0}^{(n)}\right)$. This implies $T$ is St-fa-n-continuous on $X_{1} \times X_{2} \times \ldots \times X_{n}$.

Coming to converse let us assume $T$ is St-fa-n-continuous on $X_{1} \times X_{2} \times \ldots \times X_{n}$, applying fuzzy-anti-n-continuity at $\left(x^{(1)}, x^{(2)}, \ldots, x^{(n)}\right)=\left(x_{0}^{(1)}, x_{0}^{(2)}, \ldots, x_{0}^{(n)}\right)$ for $\varepsilon=1$, there exists $\delta>0$, such that $\forall\left(x^{(1)}, x^{(2)}, \ldots, x^{(n)}\right) \in X_{1} \times X_{2} \times \ldots \times X_{n}$,

$$
N_{2}^{*}\left[T\left(x^{(1)}, x^{(2)}, \ldots, x^{(n)}\right)-T\left(x_{0}^{(1)}, x_{0}^{(2)}, \ldots, x_{0}^{(n)}\right), 1\right] \leq N_{1}^{*}\left[\left(x^{(1)}, x^{(2)}, \ldots, x^{(n)}\right)-\left(x_{0}^{(1)}, x_{0}^{(2)}, \ldots, x_{0}^{(n)}\right), \delta\right] .
$$

If $\left(x^{(1)}, x^{(2)}, \ldots, x^{(n)}\right) \neq\left(x_{0}^{(1)}, x_{0}^{(2)}, \ldots, x_{0}^{(n)}\right)$ and $\mathrm{t}>0$, putting $\left(x^{(1)}, x^{(2)}, \ldots, x^{(n)}\right)=\left(u^{(1)}, u^{(2)}, \ldots, u^{(n)}\right) t$

$$
\begin{aligned}
N_{2}^{*}\left[T\left(x^{(1)}, x^{(2)}, \ldots, x^{(n)}\right), t\right]=N_{2}^{*}\left[T\left(\left(u^{(1)}, u^{(2)}, \ldots, u^{(n)}\right) t\right), t\right]=N_{2}^{*}\left[t T\left(u^{(1)}, u^{(2)}, \ldots, u^{(n)}\right), t\right] \\
=N_{2}^{*}\left[T\left(u^{(1)}, u^{(2)}, \ldots, u^{(n)}\right), 1\right] \leq N_{1}^{*}\left[\left(u^{(1)}, u^{(2)}, \ldots, u^{(n)}\right), \delta\right] \\
=N_{1}^{*}\left[\frac{\left(x^{(1)}, x^{(2)}, \ldots, x^{(n)}\right)}{t}, \delta\right]=N_{1}^{*}\left[\left(x^{(1)}, x^{(2)}, \ldots, x^{(n)}\right), t \delta\right] \\
=N_{1}^{*}\left[\left(x^{(1)}, x^{(2)}, \ldots, x^{(n)}\right), \frac{t}{1 / \delta}\right]=N_{1}^{*}\left[\left(x^{(1)}, x^{(2)}, \ldots, x^{(n)}\right), \frac{t}{M}\right],
\end{aligned}
$$

where $M=\frac{1}{\delta}$, so, $N_{2}^{*}\left[T\left(x^{(1)}, x^{(2)}, \ldots, x^{(n)}\right), t\right] \leq N_{1}^{*}\left[\left(x^{(1)}, x^{(2)}, \ldots, x^{(n)}\right), \frac{t}{M}\right]$.

If $\left(x^{(1)}, x^{(2)}, \ldots, x^{(n)}\right) \neq\left(x_{0}^{(1)}, x_{0}^{(2)}, \ldots, x_{0}^{(n)}\right)$ and $t \leq 0$, then

$$
N_{2}^{*}\left[T\left(x^{(1)}, x^{(2)}, \ldots, x^{(n)}\right), t\right]=N_{1}^{*}\left[\left(x^{(1)}, x^{(2)}, \ldots, x^{(n)}\right), \frac{t}{M}\right]=1
$$

If $\left(x^{(1)}, x^{(2)}, \ldots, x^{(n)}\right)=\left(x_{0}^{(1)}, x_{0}^{(2)}, \ldots, x_{0}^{(n)}\right)$ and $t \in R$, then

$$
T\left(x_{0}^{(1)}, x_{0}^{(2)}, \ldots, x_{0}^{(n)}\right)=\left(x_{0}^{(1)}, x_{0}^{(2)}, \ldots, x_{0}^{(n)}\right)
$$

and

if $t>0$;

$$
N_{2}^{*}\left[T\left(x_{0}^{(1)}, x_{0}^{(2)}, \ldots, x_{0}^{(n)}\right), t\right]=N_{1}^{*}\left[\left(x_{0}^{(1)}, x_{0}^{(2)}, \ldots, x_{0}^{(n)}\right), \frac{t}{M}\right]=0
$$

$$
N_{2}^{*}\left[T\left(x_{0}^{(1)}, x_{0}^{(2)}, \ldots, x_{0}^{(n)}\right), t\right]=N_{1}^{*}\left[\left(x_{0}^{(1)}, x_{0}^{(2)}, \ldots, x_{0}^{(n)}\right), \frac{t}{M}\right]=1
$$

if $t \leq 0$. Therefore $T$ is St-fa-n-bounded.

Theorem 4.7 Let $T: X_{1} \times X_{2} \times \ldots \times X_{n} \rightarrow Y_{1} \times Y_{2} \times \ldots \times Y_{n}$ be a fuzzy-anti-n-linear mapping, $X_{1}, X_{2}, \ldots, X_{n}$ and $Y_{1}, Y_{2}, \ldots, Y_{n}$ are subspaces of $\left(X, N_{1}^{*}\right),\left(Y, N_{2}^{*}\right)$ respectively. Then 
(i) $T$ is Wk-fa-n-continuous on $X_{1} \times X_{2} \times \ldots \times X_{n}$ if $T$ is Wk-fa-n-continuous at a point $\left(x_{0}^{(1)}, x_{0}^{(2)}, \ldots . ., x_{0}^{(n)}\right) \in$ $X_{1} \times X_{2} \times \ldots \times X_{n}$.

(ii) $T$ is Wk-fa-n-continuous if and only if $T$ is Wk-fa-n-bounded.

Proof. (i) Since $T$ is Wk-fa-n-continuous at $\left(x_{0}^{(1)}, x_{0}^{(2)}, \ldots . ., x_{0}^{(n)}\right) \in X_{1} \times X_{2} \times \ldots \times X_{n}$ for $\varepsilon>0, \alpha \in(0,1), \exists$ $\delta=\delta(\alpha, \varepsilon)>0$, such that $\forall\left(x^{(1)}, x^{(2)}, \ldots, x^{(n)}\right) \in X_{1} \times X_{2} \times \ldots . . \times X_{n}$

$$
\begin{aligned}
& N_{1}^{*}\left[\left(x^{(1)}, x^{(2)}, x^{(3)}, \ldots, x^{(n)}\right)-\left(x_{0}^{(1)}, x_{0}^{(2)}, x_{0}^{(3)}, \ldots, x_{0}^{(n)}\right), \delta\right] \leq 1-\alpha \\
\Rightarrow & N_{2}^{*}\left[T\left(x^{(1)}, x^{(2)}, x^{(3)}, \ldots, x^{(n)}\right)-T\left(x_{0}^{(1)}, x_{0}^{(2)}, x_{0}^{(3)}, \ldots, x_{0}^{(n)}\right), \varepsilon\right] \leq 1-\alpha
\end{aligned}
$$

taking $\left(y^{(1)}, y^{(2)}, \ldots . ., y^{(n)}\right) \in X_{1} \times X_{2} \times \ldots \times X_{n}$ and replacing $\left(x^{(1)}, x^{(2)}, \ldots . ., x^{(n)}\right)$ by $\left(x^{(1)}, x^{(2)}, \ldots, x^{(n)}\right)+\left(x_{0}^{(1)}, x_{0}^{(2)}, \ldots, x_{0}^{(n)}\right)-$ $\left(y^{(1)}, y^{(2)}, \ldots, y^{(n)}\right)$, we get

$$
\begin{gathered}
N_{1}^{*}\left[\left(x^{(1)}, x^{(2)}, \ldots, x^{(n)}\right)+\left(x_{0}^{(1)}, x_{0}^{(2)}, \ldots, x_{0}^{(n)}\right)-\left(y^{(1)}, y^{(2)}, \ldots, y^{(n)}\right)-\left(x_{0}^{(1)}, x_{0}^{(2)}, \ldots, x_{0}^{(n)}\right), \delta\right] \leq 1-\alpha \\
N_{2}^{*}\left[T\left[\left(x^{(1)}, x^{(2)}, \ldots, x^{(n)}\right)+\left(x_{0}^{(1)}, x_{0}^{(2)}, \ldots, x_{0}^{(n)}\right)-\left(y^{(1)}, y^{(2)}, \ldots, y^{(n)}\right)\right]-T\left(x_{0}^{(1)}, x_{0}^{(2)}, \ldots, x_{0}^{(n)}\right), \varepsilon\right] \leq 1-\alpha
\end{gathered}
$$

(i.e.)

$$
\begin{gathered}
N_{1}^{*}\left[\left(x^{(1)}, x^{(2)}, \ldots, x^{(n)}\right)-\left(y^{(1)}, y^{(2)}, \ldots, y^{(n)}\right), \delta\right] \leq 1-\alpha \\
N_{2}^{*}\left[T\left(x^{(1)}, x^{(2)}, \ldots, x^{(n)}\right)-T\left(y^{(1)}, y^{(2)}, \ldots, y^{(n)}\right), \varepsilon\right] \leq 1-\alpha .
\end{gathered}
$$

Since $\left(y^{(1)}, y^{(2)}, \ldots . ., y^{(n)}\right) \in X_{1} \times X_{2} \times \ldots \times X_{n}$ is arbitrary, $T$ is Wk-fa-n-continuous on $X_{1} \times X_{2} \times \ldots \times X_{n}$.

(ii) Now we assume $T$ is Wk-fa-n-bounded. Thus for any $\alpha \in(0,1)$ there exists $M_{\alpha}>0$, such that $\forall t \in R$ and for all $\left(x^{(1)}, x^{(2)}, \ldots, x^{(n)}\right) \in X_{1} \times X_{2} \times \ldots \times X_{n}$, we have

$$
N_{1}^{*}\left[\left(x^{(1)}, x^{(2)}, \ldots, x^{(n)}\right), \frac{t}{M}\right] \leq 1-\alpha \Rightarrow N_{2}^{*}\left[T\left(x^{(1)}, x^{(2)}, \ldots, x^{(n)}\right), t\right] \leq 1-\alpha .
$$

Therefore

$$
\begin{aligned}
& N_{1}^{*}\left[\left(x^{(1)}, x^{(2)}, \ldots, x^{(n)}\right)-\left(\theta^{(1)}, \theta^{(2)}, \ldots, \theta^{(n)}\right), \frac{t}{M}\right] \leq 1-\alpha \\
\Rightarrow & N_{2}^{*}\left[T\left(x^{(1)}, x^{(2)}, \ldots, x^{(n)}\right)-T\left(\theta^{(1)}, \theta^{(2)}, \ldots, \theta^{(n)}\right), t\right] \leq 1-\alpha
\end{aligned}
$$

(i.e.)

$$
\begin{aligned}
& N_{1}^{*}\left[\left(x^{(1)}, x^{(2)}, \ldots, x^{(n)}\right)-\left(\theta^{(1)}, \theta^{(2)}, \ldots, \theta^{(n)}\right), \frac{\varepsilon}{M_{\alpha}}\right] \leq 1-\alpha \\
\Rightarrow & N_{2}^{*}\left[T\left(x^{(1)}, x^{(2)}, \ldots, x^{(n)}\right)-T\left(\theta^{(1)}, \theta^{(2)}, \ldots, \theta^{(n)}\right), \varepsilon\right] \leq 1-\alpha
\end{aligned}
$$

(i.e.)

$$
\begin{aligned}
& N_{1}^{*}\left[\left(x^{(1)}, x^{(2)}, \ldots, x^{(n)}\right)-\left(\theta^{(1)}, \theta^{(2)}, \ldots, \theta^{(n)}\right), \delta\right] \leq 1-\alpha \\
\Rightarrow & N_{2}^{*}\left[T\left(x^{(1)}, x^{(2)}, \ldots, x^{(n)}\right)-T\left(\theta^{(1)}, \theta^{(2)}, \ldots, \theta^{(n)}\right), \varepsilon\right] \leq 1-\alpha
\end{aligned}
$$

where $\frac{\varepsilon}{M_{\alpha}}=\delta$. Therefore $T$ is Wk-fa-n-continuous at $\left(x_{0}^{(1)}, x_{0}^{(2)}, \ldots . ., x_{0}^{(n)}\right)$, which implies $T$ is Wk-fa-n-continuous on $X_{1} \times X_{2} \times \ldots \times X_{n}$.

Coming to converse let us assume $T$ is Wk-fa-n-continuous on $X_{1} \times X_{2} \times \ldots \times X_{n}$, applying continuity of $\mathrm{T}$ at $\left(\theta^{(1)}, \theta^{(2)}, \ldots, \theta^{(n)}\right)$ and take $\varepsilon=1$, we have $\forall \alpha \in(0,1) \exists \delta(\alpha, 1)>0$, such that $\forall\left(x^{(1)}, x^{(2)}, \ldots, x^{(n)}\right) \in X_{1} \times X_{2} \times \ldots \times X_{n}$, (i.e.)

$$
\begin{aligned}
& N_{1}^{*}\left[\left(x^{(1)}, x^{(2)}, \ldots, x^{(n)}\right)-\left(\theta^{(1)}, \theta^{(2)}, \ldots, \theta^{(n)}\right), \delta\right] \leq 1-\alpha \\
\Rightarrow & N_{2}^{*}\left[T\left(x^{(1)}, x^{(2)}, \ldots, x^{(n)}\right)-T\left(\theta^{(1)}, \theta^{(2)}, \ldots, \theta^{(n)}\right), 1\right] \leq 1-\alpha
\end{aligned}
$$

(i.e.)

$$
N_{1}^{*}\left[\left(x^{(1)}, x^{(2)}, \ldots, x^{(n)}\right), \delta\right] \leq 1-\alpha \Rightarrow N_{2}^{*}\left[T\left(x^{(1)}, x^{(2)}, \ldots, x^{(n)}\right), 1\right] \leq 1-\alpha .
$$

If $\left(x^{(1)}, x^{(2)}, \ldots, x^{(n)}\right) \neq\left(\theta^{(1)}, \theta^{(2)}, \ldots, \theta^{(n)}\right)$ and $t>0$, putting $\left(x^{(1)}, x^{(2)}, \ldots ., x^{(n)}\right)=\frac{\left(u^{(1)}, u^{(2)}, \ldots, u^{(n)}\right.}{t}$

$$
N_{1}^{*}\left(\frac{\left(u^{(1)}, u^{(2)}, \ldots ., u^{(n)}\right)}{t}, \delta\right) \leq 1-\alpha \Rightarrow N_{2}^{*}\left(T\left(\frac{\left(u^{(1)}, u^{(2)}, \ldots ., u^{(n)}\right)}{t}\right), 1\right) \leq 1-\alpha
$$


(i.e.)

$$
N_{1}^{*}\left(\left(u^{(1)}, u^{(2)}, \ldots ., u^{(n)}\right), t \delta\right) \leq 1-\alpha \Rightarrow N_{2}^{*}\left(T\left(\frac{\left(u^{(1)}, u^{(2)}, \ldots ., u^{(n)}\right)}{t}\right), 1\right) \leq 1-\alpha
$$

(i.e.)

$$
N_{1}^{*}\left(\left(u^{(1)}, u^{(2)}, \ldots ., u^{(n)}\right), \frac{t}{M_{\alpha}}\right) \leq 1-\alpha \Rightarrow N_{2}^{*}\left(T\left(\frac{\left(u^{(1)}, u^{(2)}, \ldots ., u^{(n)}\right)}{t}\right), 1\right) \leq 1-\alpha
$$

where $M_{\alpha}=\frac{1}{\delta(\alpha, 1)}$. So

$$
\begin{gathered}
N_{1}^{*}\left[t\left(x^{(1)}, x^{(2)}, \ldots ., x^{(n)}\right), \frac{t}{M_{\alpha}}\right] \leq 1-\alpha \Rightarrow N_{2}^{*}\left[T\left(x^{(1)}, x^{(2)}, \ldots ., x^{(n)}\right), 1\right] \leq 1-\alpha \\
N_{1}^{*}\left[\left(x^{(1)}, x^{(2)}, \ldots ., x^{(n)}\right), \frac{t}{M_{\alpha}}\right] \leq 1-\alpha \Rightarrow N_{2}^{*}\left[T\left(\frac{\left(x^{(1)}, x^{(2)}, \ldots, x^{(n)}\right)}{t}\right), 1\right] \leq 1-\alpha \\
N_{1}^{*}\left[\left(x^{(1)}, x^{(2)}, \ldots ., x^{(n)}\right), \frac{t}{M_{\alpha}}\right] \leq 1-\alpha \Rightarrow N_{2}^{*}\left[T\left(\left(x^{(1)}, x^{(2)}, \ldots ., x^{(n)}\right)\right), t\right] \leq 1-\alpha,
\end{gathered}
$$

where $M_{\alpha}=\frac{1}{\delta(\alpha, 1)}$. If $\left(x^{(1)}, x^{(2)}, \ldots, x^{(n)}\right) \neq\left(\theta^{(1)}, \theta^{(2)}, \ldots, \theta^{(n)}\right)$ and $t \leq 0$,

$$
N_{1}^{*}\left[\left(x^{(1)}, x^{(2)}, \ldots ., x^{(n)}\right), \frac{t}{M_{\alpha}}\right]=N_{2}^{*}\left[T\left(\left(x^{(1)}, x^{(2)}, \ldots ., x^{(n)}\right)\right), t\right]=1 \text { for any } M_{\alpha}>0 .
$$

If $\left(x^{(1)}, x^{(2)}, \ldots, x^{(n)}\right)=\left(\theta^{(1)}, \theta^{(2)}, \ldots, \theta^{(n)}\right)$, then for $M_{\alpha}>0$,

$$
\begin{aligned}
& N_{1}^{*}\left[\left(x^{(1)}, x^{(2)}, \ldots ., x^{(n)}\right), \frac{t}{M_{\alpha}}\right]=N_{2}^{*}\left[T\left(\left(x^{(1)}, x^{(2)}, \ldots ., x^{(n)}\right)\right), t\right]=0, \text { if } t>0, \\
& N_{1}^{*}\left[\left(x^{(1)}, x^{(2)}, \ldots ., x^{(n)}\right), \frac{t}{M_{\alpha}}\right]=N_{2}^{*}\left[T\left(\left(x^{(1)}, x^{(2)}, \ldots ., x^{(n)}\right)\right), t\right]=1, \text { if } t \leq 0 .
\end{aligned}
$$

Therefore $T$ is Wk-fa-n-bounded.

\section{References}

Bag, T., \& Samanta, T. K. (2003). Finite Dimensional Fuzzy normed linear spaces. The journal of Fuzzy Mathematics, 11(3), 687-705.

Felbin, C. (1993). The Completion of Fuzzy normed linear space. Journal of Mathematical Analysis and Applications, 174(2), 428-440. http://dx.doi.org/10.1006/jmaa.1993.1128

Jebril, I. H., \& Samanta, T. K. (2010). Fuzzy Anti normed spaces. Journal of Mathematics and Technology, 1(1), 66-77.

Katsaras, A. K. (1984). Fuzzy Topological Vector spaces. Fuzzy Sets and Systems, 12, 143-154. http://dx.doi.org/10.1016/0165-0114(84)90034-4

Meenakshi, A. K., \& Cokilavanya, R. (2001). On Fuzzy 2-normed linear spaces. The journal of Fuzzy Mathematics, 9(2), 345-351.

Narayan, A. L., \& Vijayabalaji, S. (2005). Fuzzy n-normed linear space. International Journal of Mathematics and Mathematical Sciences, 24, 3963-3977. http://dx.doi.org/10.1155/IJMMS.2005.3963

Reddy, B. S. (2011a). Fuzzy anti 2-normed linear space. Journal of Mathematics Research, 3(2), 137-144. http://dx.doi.org/10.5539/jmr.v3n2p137

Reddy, B. S. (2011b). Fuzzy anti n-normed linear space. Journal of Mathematics and Technology, 2(1), 14-26.

Sinha, P., Mishra, D., \& Lal, G. (2011). Fuzzy anti 2-continuos linear operator. Journal of Mathematics and Technology, 2(4), 9-19.

Sinha, P., Mishra, D., \& Lal, G. (2012). Fuzzy anti 2-bounded linear operator. International Journal of Mathematics Research, 4(1), 71-84. 


\section{Copyrights}

Copyright for this article is retained by the author(s), with first publication rights granted to the journal.

This is an open-access article distributed under the terms and conditions of the Creative Commons Attribution license (http://creativecommons.org/licenses/by/3.0/). 